\title{
The role of miRNA, IncRNA and circRNA in the development of intervertebral disk degeneration (Review)
}

\author{
JIAN JIANG $^{1 *}$, YUEFENG SUN ${ }^{2 *}$, GAORAN XU ${ }^{3}$, HONG WANG ${ }^{2}$ and LING WANG ${ }^{4}$ \\ ${ }^{1}$ Department of Minimally Invasive Spine Surgery, The Third Affiliated Hospital of Southern Medical University, \\ Guangzhou, Guangdong 510000; ${ }^{2}$ Department of Spine Surgery, The First Affiliated Hospital of Dalian Medical University; \\ ${ }^{3}$ Department of General Surgery, The Second Hospital of Dalian Medical University; ${ }^{4}$ Department of Oncology Surgery, \\ The First Affiliated Hospital of Dalian Medical University, Dalian, Liaoning 116000, P.R. China
}

Received December 23, 2019; Accepted June 5, 2020

DOI: $10.3892 / \mathrm{etm} .2021 .9987$

\begin{abstract}
Intervertebral disc degeneration (IVDD) is a degenerative musculoskeletal disorder with multiple causative factors, such as age, genetics, mechanics and life style. IVDD contributes to non-specific lower back pain (NLBP), which is a globally prevalent and debilitating musculoskeletal disorder. NLBP has a substantial impact on medical resources and creates an economic burden for the public. Dysregulated phenotypes of nucleus pulposus (NP) cells and endplate chondrocytes, such as proliferation, senescence and apoptosis, along with aberrant expression of extracellular matrix components, including type II collagen and aggrecan, are involved in the pathological process of IVDD. Evidence indicates that non-coding RNAs, mainly microRNAs (miRNAs), long non-coding RNAs (lncRNAs) and circular RNAs (circRNAs), play a vital role in the development of IVDD. In the present review, the potential molecular mechanisms of miRNAs, lncRNAs and circRNAs in the initiation and progression of IVDD were described based on the latest literature. Furthermore, ways to influence the functions of NP cells and endplate chondrocytes in IVDD
\end{abstract}

Correspondence to: Professor Hong Wang, Department of Spine Surgery, The First Affiliated Hospital of Dalian Medical University, 222 Zhongshan Road, Dalian, Liaoning 116000, P.R. China

E-mail: wanghong@dmu.edu.cn

Professor Ling Wang, Department of Oncology Surgery, The First Affiliated Hospital of Dalian Medical University, 222 Zhongshan Road, Dalian, Liaoning 116000, P.R. China

E-mail: 516245530@qq.com

${ }^{*}$ Contributed equally

Abbreviations: ADAMTS, A disintegrin and metalloproteinase with thrombospondin motifs; GAS1, growth arrest specific gene 1; HOTAIR, homeobox transcript antisense intergenic RNA; MMP, matrix metalloprotein; RT-qPCR, reverse transcription-quantitative PCR; TNF- $\alpha$, tumor necrosis factor- $\alpha$

Key words: non-coding RNA, intervertebral disc degeneration, apoptosis, proliferation, extracellular matrix-degradation, senescence were also summarized. The presented insights suggested that non-coding RNAs may function as potential targets for the treatment of IVDD.

\section{Contents \\ 1. Introduction \\ 2. miRNA in IVDD \\ 3. IncRNAs in IVDD \\ 4. circRNAs in IVDD \\ 5. Conclusions}

\section{Introduction}

Non-specific low back pain (NLBP) is a prevalent and debilitating musculoskeletal disorder that affects people globally (1). NLBP causes a substantial burden on medical resources and the economy (2-4). It is estimated that the medical costs of NLBP in the USA alone are $\sim \$ 253$ billion per year (5). Efforts have been made by clinicians and researchers to investigate the pathogenesis of NLBP and develop effective treatment strategies. Increasing evidence suggests that one of the major causes of NLBP is intervertebral disc degeneration (IVDD) (6), which can be caused by inflammatory factors (7), genetic factors (8), aging (9), intervertebral instability (10) and metabolic disorders (11). Currently, the underlying molecular mechanisms between these factors and IVDD have not yet been investigated. It is well known that the IVDD is composed of the inner nucleus pulposus (NP); a proteoglycan-rich gelatinous substance, the outer annulus fibrosus, as well as the upper and lower cartilage endplates (CEP) (12) (Fig. 1). NP cells play an important role in secreting extracellular matrix (ECM) components, such as type II collagen and aggrecan, in addition to retaining water (13). CEP, a crucial nutrition and metabolic exchange channel, maintains the balance between catabolism and anabolism within IVDD $(14,15)$. Therefore, dysfunction of NP cells and CEP cells, including apoptosis, senescence and abnormal cell proliferation, may cause an imbalance between catabolism and anabolism, which is known to be involved in the pathology of IVDD $(16,17)$. 
Non-coding RNAs form a large segment of RNA molecules that are transcribed from DNA, but lack the potential to be translated into proteins or peptides (18). Non-coding RNAs include short hairpin RNA, small interfering RNA, antisense RNA, microRNA (miRNA), long non-coding RNA (lncRNA), circular RNA (circRNA) and extracellular RNAs (18-25). Increasing evidence suggests that miRNAs, lncRNAs and circRNAs have a vital regulatory function in the pathological process of several diseases, such as cancer (26-31), cardiac disease (32-35) and IVDD (36-41). The structures of miRNAs, lncRNAs and circRNAs are presented in Fig. 2 miRNAs, a class of small non-coding RNAs that are 19-25 nucleotides in length, suppress gene expression by directly binding to the 3'-untranslated regions (UTR), 5'-UTR and coding sequence regions of their target mRNAs, leading to translational repression and/or cleavage (42). This direct binding to 3'-UTR is the primary method by which miRNAs regulate target genes (43-49). Conversely, lncRNAs are the largest non-coding RNAs (>200 nucleotides in length) without an open reading frame (50). IncRNAs exert physiological functions by modulating gene expression at multiple levels, including DNA methylation, recruitment of transcriptional factors, miRNA sponges and protein-protein interactions (50-55). Unlike the linear structures of miRNAs and lncRNAs, circRNAs are characterized by covalently closed single-stranded loop structures without free 3 ' and 5 ' ends (56). This structure hinders the digestion by ribonucleases $\mathrm{R}$ and exonucleases (56-58). circRNAs are produced by a precursor mRNA back-splicing mechanism (59). Furthermore, they are widely expressed in eukaryotes with cell type- and tissue-specific patterns, acting as competing endogenous RNAs (ceRNAs) and transcriptional regulators $(29,60,61)$. The present review article provides an overview of the role of miRNAs, IncRNAs and circRNAs in the pathological process of IVDD based on recent studies, in an attempt to clarify the diagnosis and treatment of IVDD.

\section{2. miRNA in IVDD}

Evidence indicates that abnormal proliferation of NP cells and formation of cell clusters are implicated in IVDD pathogenesis (45). Li et al (62) demonstrated that the expression of miR-184 was positively associated with Pfirrmann scores (63) and upregulated in degenerative NP samples compared with that in normal NP samples. Furthermore, luciferase assays from the same study indicated that growth arrest specific gene 1 (GAS1) is a target of miR-184, and degenerative NP tissues present low expression of GAS1 compared with normal NP samples (59). Functionally, overexpression of miR-184 can promote abnormal proliferation and cluster formation of NP cells by inducing AKT phosphorylation, which plays an important role in the development of IVDD (62). However, unequivocal evidence demonstrates that apoptosis exists in diverse biological processes, including IVDD (64). The expression levels of miR-138-5p and miR-494 in degenerated NP tissues compared with normal tissue controls, and their effects on apoptosis were investigated by Wang et al and Wang et al $(43,65)$. The aim of their research was to identify the role of miRNAs in the pathogenesis of IVDD. A total of two signaling pathways (miR-138-5p/SIRT1/PTEN/PI3K/Akt and miR-494/JunD) were discovered through gain- and loss-of-function studies. The

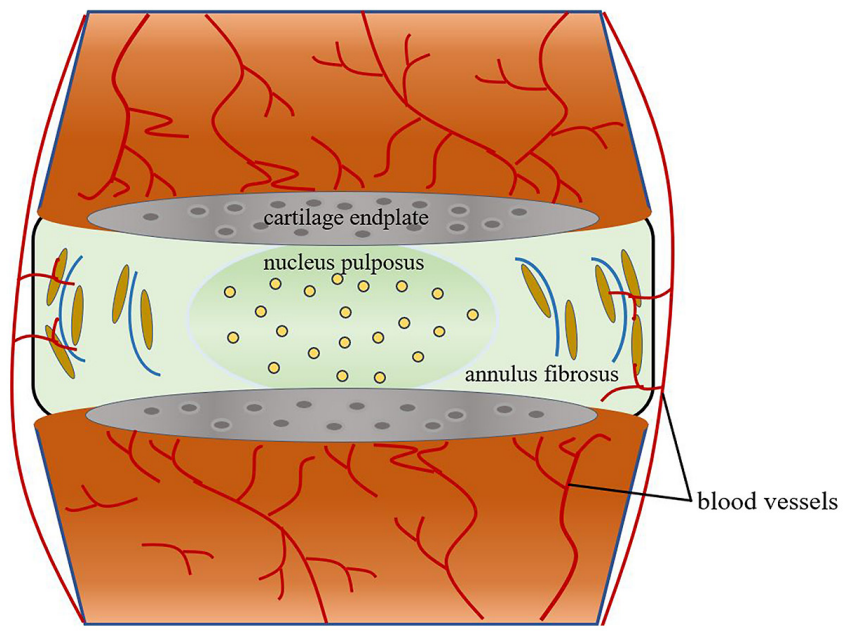

Figure 1. Schematic diagram of IVD structure. The IVD consists of the nucleus pulposus in the center, the surrounding annulus fibrosus and the upper and lower cartilage endplates. IVD, intervertebral disc.

results demonstrated that miR-138-5p (43) and miR-494 (65) promote tumor necrosis factor- $\alpha$ (TNF- $\alpha)$-induced apoptosis of NP cells in IVDD by targeting silent mating type information regulation 2 homolog-1 and the transcription factor jun-D via the PTEN/PI3K/AKT signaling pathway and cytochrome $c$ apoptotic signaling, respectively.

Recent studies have reported an imbalance between anabolism and catabolism of ECM in the development of IVDD, predominantly due to excessive ECM degradation. Wang et al and Wang et al $(37,66)$ investigated whether miR-210 and miR-21 facilitate the degradation of ECM components, such as type II collagen and aggrecan within NP tissues. The results indicated that the expression levels of miR-210 and miR-21 are significantly upregulated in degenerated NP specimens compared with healthy controls. Furthermore, miR-210 and miR-21 expression exhibited a positive association with the grade of IVDD disease, using miRNA microarray and reverse transcription-quantitative (RT-q)PCR validation assays. Knockdown and overexpression of miR-210/miR-21 were followed by observation of downstream target genes and ECM-related gene expression compared with the control group. The aforementioned gain- and loss-of-function studies demonstrated that miR-210 and miR-21 promote ECM degradation by suppressing autophagy, targeting both the autophagy-related protein 7 and the PTEN/AKT/mTOR signaling pathway in human NP cells. Conversely, several miRNAs are downregulated in degenerative NP tissues, indicating that miRNAs may exert a protective effect on normal NP tissues against degeneration (45). Studies have indicated that 51 miRNAs are differentially expressed in degenerated intervertebral discs compared with normal intervertebral discs (67). Of these, downregulation of miR-127-5p, miR-193a-3p, miR-133a and miR-98 induce loss of ECM components by targeting matrix metalloproteinase (MMP)-13, MMP-14, MMP-9 and interleukin-6, respectively (68-71). Other miRNAs that have not been studied further may be found to have no differential expression using (RT-q)PCR.

The dysregulation of cell proliferation, matrix hardness and ECM degradation of CEP are also involved in the progression 


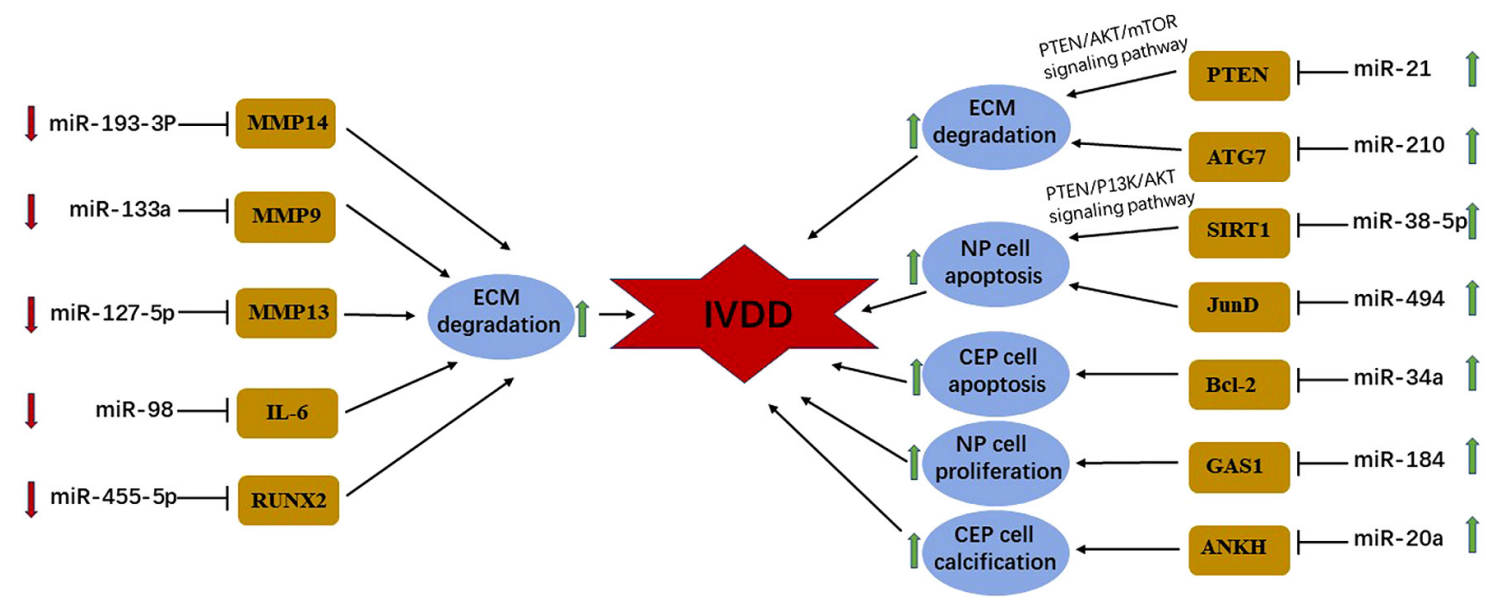

Figure 2. Functional mechanism of upregulated and downregulated expression of miRNAs in IVDD. Upregulated miRNAs (miR-21, -210, -38-5P, -494, $-34 a,-184$ and -20a) and downregulated miRNAs (miR-193-3P, -133a, -127-5p, -98 and -455-5p) in a degenerative IVD facilitate the progression of IVDD by promoting ECM degradation, NP cell apoptosis and proliferation and CEP cell apoptosis, and calcification by regulating corresponding target genes. miRNA/miR, microRNA; IVD, intervertebral disc; IVDD, intervertebral disc degeneration; ECM, extracellular matrix; NP, nucleus pulposus; CEP, cartilage endplates; MMP, matrix metalloproteinase; IL-6, interleukin-6; RUNX2, Runt-related transcription factor 2; ATG7, autophagy-related protein 7; SIRT1, silent mating type information regulation 2 homolog-1; JunD, transcription factor jun-D; Bcl-2, B-cell lymphoma-2; GAS1, growth arrest specific gene 1; ANKH, ankylosis protein homolog.

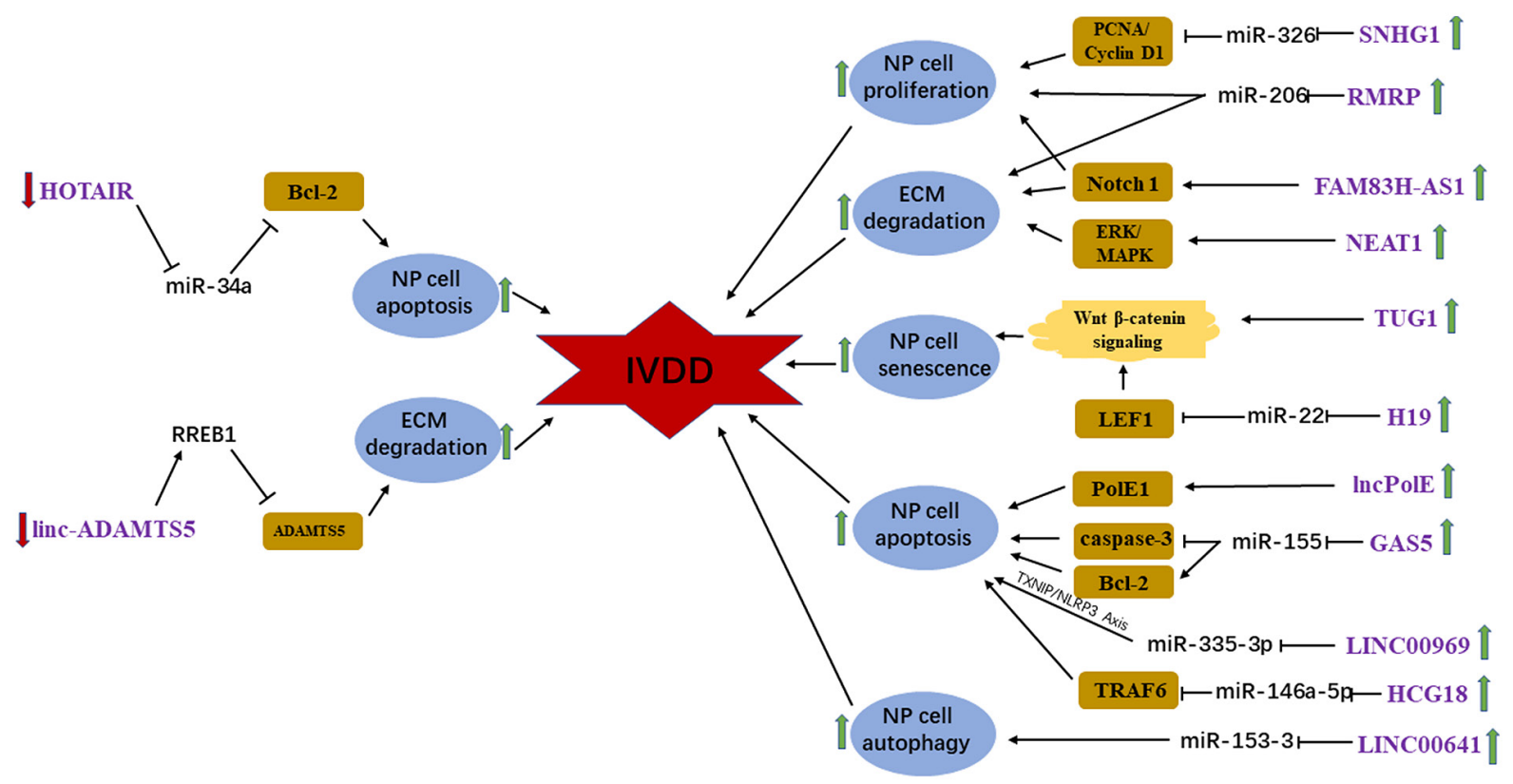

Figure 3. Functional mechanism of upregulated and downregulated expression of lncRNAs in IVDD. Upregulated lncRNAs (SNHG1, RMRP, FAM83H-AS1, NEAT1, TUG1, H19, IncPolE, GAS5, LINC00969, HCG18 and LINC00641) and downregulated lncRNAs (HOTAIR and linc-ADAMTS5) in a degenerative IVD facilitate the development of IVDD by enhancing ECM degradation and proliferation, senescence, apoptosis and autophagy of NP cells by modulating different downstream targets. IncRNA, long non-coding RNA; IVDD, intervertebral disc degeneration; ECM, extracellular matrix; NP, nucleus pulposus; miR, microRNA; SNHG1, small nucleolar RNA host gene 1; RMRP, RNA component of mitochondrial RNA processing endoribonuclease; FAM83H-AS1, IQ motif and ankyrin repeat containing; NEAT1, nuclear paraspeckle assembly transcript 1; TUG1, taurine upregulated gene 1; H19, H19 imprinted maternally expressed transcript; LINC00969, long intergenic non-protein coding RNA 969; HCG18, HLA complex group 18; LINC00641, long intergenic non-protein coding RNA 641; HOTAIR, homeobox transcript antisense intergenic RNA; GAS5, growth arrest specific 5; linc, long intergenic non-protein coding RNA; ADAMTS5, A disintegrin and metalloproteinase with thrombospondin motif 5; PCNA, proliferating cell nuclear antigen; MAPK, mitogen-activated protein kinase; LEF1, lymphoid enhancer binding factor 1; PolE1, DNA polymerase E catalytic subunit A; TRAF6, TNF receptor-associated factor 6; RREB1, ras-responsive element-binding protein 1; TXNIP, thioredoxin interacting protein; NLRP3, NLR family pyrin domain containing 3.

of IVDD (72). Chen et al (72) performed a RT-qPCR analysis, which verified that the expression of miR-34a is markedly elevated in the CEP samples obtained from patients with IVDD compared with samples of healthy donors. Functionally, apoptosis and proliferation of CEP cells are facilitated by upregulating miR-34a through targeting Bcl-2. Liu et al and Xiao et al $(73,74)$ investigated the underlying molecular mechanisms of miR-20a and miR-455-5p in the pathogenesis of IVDD. The results demonstrated that matrix stiffness and ECM loss of CEP are positively associated with the degree of IVDD. Overexpression of miR-20a, which is upregulated in degenerative CEP tissues, accelerates the development of IVDD 


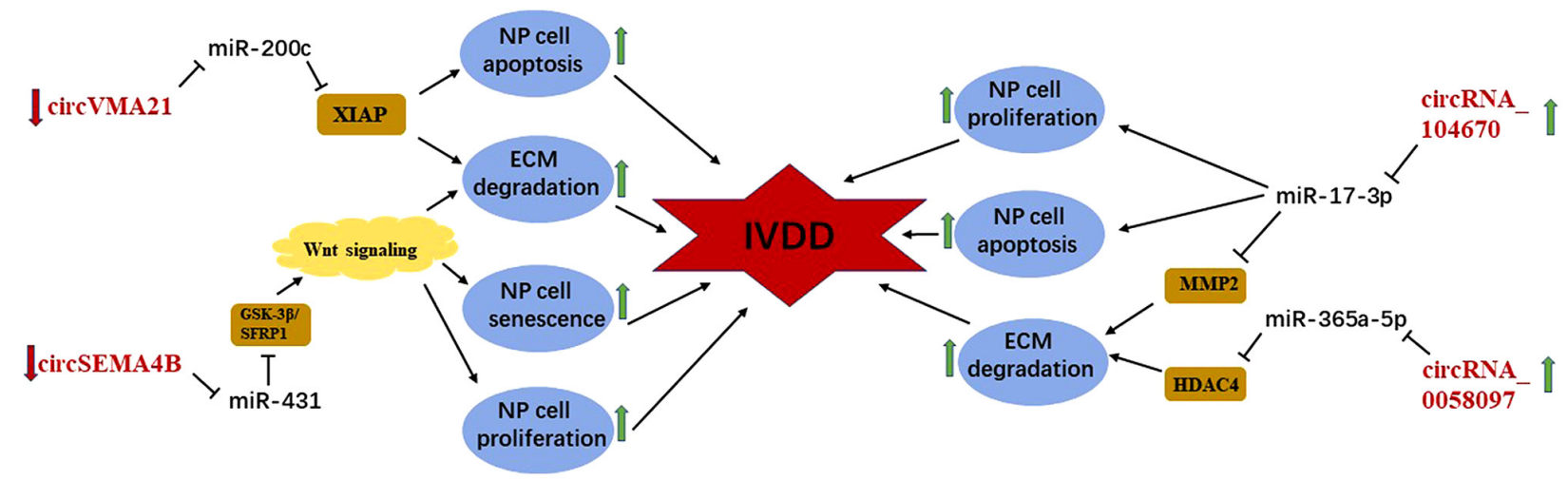

Figure 4. Functional mechanism of upregulated and downregulated expression of circRNAs in IVDD. Upregulated circRNAs (circRNA-10467 and circRNA-0058079) and downregulated circRNAs (circVMA21 and circSEMA4B) in a degenerative IVD accelerate the pathological process of IVDD by promoting ECM degradation and proliferation, apoptosis and senescence of NP cells by sponging diverse miRs and various signaling pathways. circRNA, circular RNA; IVD, intervertebral disc; IVDD, intervertebral disc degeneration; miR, microRNA; ECM, extracellular matrix; NP, nucleus pulposus; MMP2, matrix metalloprotein 2; HDAC4, histone deacetylase 4; XIAP, X-linked inhibitor-of-apoptosis protein; GSK-3 $\beta$, glycogen synthase kinase 3 $\beta$; SFRP1, secreted frizzled related protein 1 .

and facilitates calcification in CEP cells resulting in matrix stiffness by suppressing the expression of ankylosis protein homolog. Similarly, enforced expression of miR-455-5p, which is downregulated in degenerative CEP samples, promotes the progression of IVDD and increases ECM loss by targeting Runt-related transcription factor 2 (73). Based on these findings, it is speculated that miRNA may serve as a potential novel therapeutic target for IVDD (Fig. 3).

\section{IncRNAs in IVDD}

Evidence indicates that lncRNAs are involved in the pathological process of IVDD and play a key role in relevant signaling axes (75). Previous studies have demonstrated that the ectopic expression of homeobox transcript antisense intergenic RNA (HOTAIR), lncPolE, growth arrest specific 5, long intergenic non-protein coding RNA 969 and HLA complex group 18 (HCG18) contributes to initiation of IVDD by inducing apoptosis of NP cells through diverse signaling pathways (76-80). Of theselncRNAs,HOTAIR is downregulated in degenerative NP samples and inhibits TNF- $\alpha$-induced apoptosis of NP cells by regulating Bcl-2 through sponging miR-34a (76). However, the other aforementioned lncRNAs are markedly upregulated in degenerative NP tissues and promote apoptosis of NP cells by targeting DNA polymerase E catalytic subunit A, miR-155, miR-335-3p and miR-146a-5p, respectively (77-80). HCG18 increases the rate of apoptosis of NP cells and inhibits the proliferation of NP cells through the miR-146a-5p/TNF receptor-associated factor $6 / \mathrm{NF} \kappa \mathrm{B}$ axis (80). Tan et al, Wang et al and Wei et al $(39,81,82)$ first demonstrated that ectopic expression of small nucleolar RNA host gene 1, RNA component of mitochondrial RNA processing endoribonuclease (RMRP) and IQ motif and ankyrin repeat containing (FAM83H-AS1), which are substantially upregulated in IVDD samples compared with control samples, promote the progression of IVDD by enhancing NP cell proliferation. Mechanistically, they suppress the expression of miR-326, miR-206 and Notch1 to promote NP cell proliferation. Furthermore, RMRP and FAM83H-AS1 also demonstrate the ability to modulate the

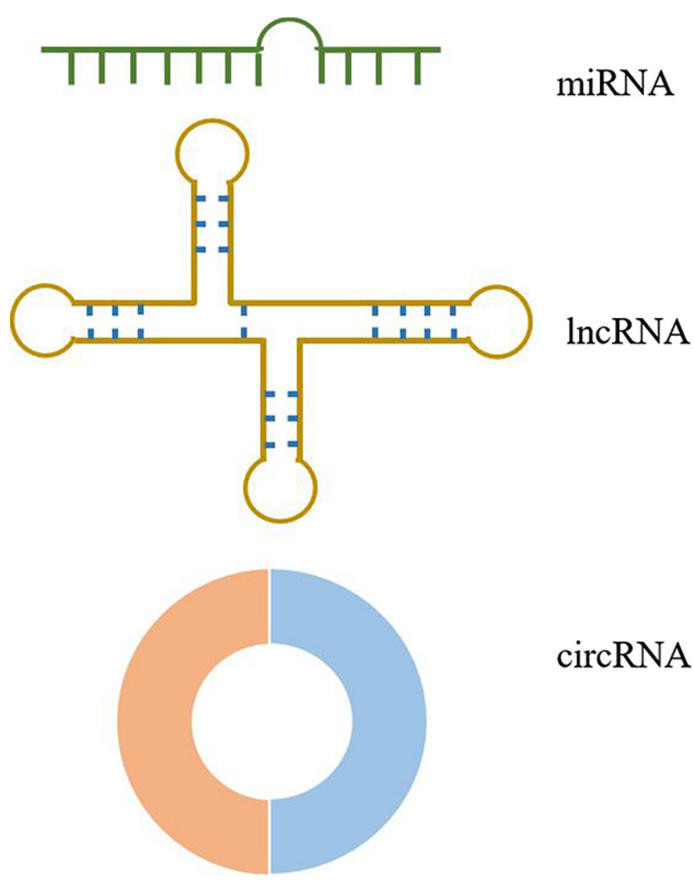

Figure 5. Schematic structures of miRNA, lncRNA and circRNA. miRNAs, a class of small non-coding RNAs of 19-25 nucleotides in length, suppress gene expression by binding directly to the 3'-UTR, 5'-UTR and coding sequence regions of their target mRNAs, leading to translational repression and/or cleavage. 1 ncRNAs are the largest non-coding RNAs (>200 nucleotides in length) without an open reading frame and exhibit physiological functions by modulating gene expressions at multiple levels including DNA methylation, recruitment of transcriptional factors, miRNA sponges and protein-protein interactions. circRNAs are characterized by covalently closed single-stranded loop structures without free 3 ' and $5^{\prime}$ ends and the most frequently described function of circRNAs is to bind miRNAs, preventing them from binding to their canonical mRNA target genes (sponge effect). miRNA, microRNA; lncRNA, long non-coding RNA; circRNA, circular RNA; UTR, untranslated regions.

expression of ECM components, including type II collagen and aggrecan $(39,81,82)$. Ruan et al and Wang et al $(83,84)$ confirmed that nuclear paraspeckle assembly transcript 1 (NEAT1) and long intergenic non-protein coding RNA (linc)-A disintegrin and MMP with thrombospondin motifs 
Table I. Characteristics of miRNAs involved in the process of intervertebral disc degeneration.

\begin{tabular}{lclllll}
\hline miRNA & Specimen & Expression & Target & \multicolumn{1}{c}{ Function } & Author, year & (Refs.) \\
\hline miR-210 & NP & Up & ATG7 & ECM-degradation $\uparrow$ & Wang et al, 2017 & $(37)$ \\
miR-138-5p & NP & Up & SIRT1 & Apoptosis $\uparrow$ & Wang et al, 2016 & $(43)$ \\
miR-184 & NP & Up & GAS1 & Proliferation $\uparrow$ & Li et al, 2017 & $(62)$ \\
miR-494 & NP & Up & JunD & Apoptosis $\uparrow$ & Wang et al, 2016 & $(65)$ \\
miR-21 & NP & Up & PTEN & ECM-degradation $\uparrow$ & Wang et al, 2018 & $(66)$ \\
miR-193a-3p & NP & Down & MMP-14 & ECM-degradation $\downarrow$ & Wang et al, 2016 & $(68)$ \\
miR-133a & NP & Down & MMP-9 & ECM-degradation $\downarrow$ & Xu et al, 2016 & $(69)$ \\
miR-127-5p & NP & Down & MMP-13 & ECM-degradation $\downarrow$ & Hua et al, 2017 & $(70)$ \\
miR-98 & NP & Down & IL-6 & ECM-degradation $\downarrow$ & Ji et al, 2016 & $(71)$ \\
miR-34a & CEP & Up & Bcl-2 & Apoptosis $\uparrow$ & Chen et al, 2016 & $(72)$ \\
miR-20a & CEP & Up & ANKH & Calcification $\uparrow$ & Liu et al, 2016 & $(73)$ \\
miR-455-5p & CEP & Down & RUNX2 & ECM-degradation $\downarrow$ & Xiao et al, 2018 & $(74)$ \\
\hline
\end{tabular}

$\uparrow$, promotion; $\downarrow$, inhibition. miRNA/miR, microRNA; ATG7, autophagy-related protein 7; SIRT1, silent mating type information regulation 2 homolog-1; GAS1, growth arrest specific gene 1; JunD, transcription factor jun-D; MMP, matrix metalloprotein; IL-6, interleukin-6; Bcl-2, B-cell lymphoma-2; ANKH, ankylosis protein homolog; RUNX2, Runt-related transcription factor 2; ECM, extracellular matrix; NP, nucleus pulposus; CEP, cartilage endplates.

(ADAMTS)5 play crucial roles in the progression of IVDD by regulating the balance between synthesis and degradation of the ECM. However, the expression levels of NEAT1 and linc-ADAMTS5 are different in NP tissues isolated from patients with IVDD. In IVDD, NEAT1 and linc-ADAMTS5 are notably upregulated and downregulated, respectively. Functionally, NEAT1 promotes ECM degradation by upregulating MMP-13 and ADAMTS4 (genes encoding ECM-associated enzymes), and downregulating collagen II and aggrecan through the ERK/mitogen-activated protein kinase signaling pathway. Linc-ADAMTS5 interacts with Ras-responsive element-binding protein 1 to suppress the degradation of ECM and inhibit the expression of ADAMTS5 $(83,84)$. Notably, it was identified that two different lncRNAs, taurine upregulated gene 1 (TUG1) and H19 imprint maternally expressed transcript (H19), modulate NP cell senescence, apoptosis and ECM synthesis through the Wnt/ $\beta$-catenin signaling pathway $(85,86)$. Functionally, TUG1 and $\mathrm{H} 19$, which are both upregulated in degenerative NP tissues, promote NP cell senescence, apoptosis and ECM degradation by targeting $\mathrm{Wnt} / \beta$-catenin and miR-22, respectively $(85,86)$. A recent study by Wang et al (87) focused on the role of autophagy in the pathogenesis of IVDD and demonstrated that the long intergenic non-protein coding RNA 641, which is markedly upregulated in NP samples obtained from patients with IVDD compared with controls, regulate the development of IVDD by inducing autophagic cell death through targeting miR-153-3p and autophagy-related gene 5. In addition, some treatments can be used to target lncRNAs in IVDD, such as silencing of lncRNAs, locked nucleic acid GapmeRs, small molecule inhibitors, antisense nucleotides and zinc-finger nucleases (88). IncRNAs may represent potential effective novel targets for the treatment of IVDD (Fig. 4).

\section{4. circRNAs in IVDD}

circRNAs are involved in the regulation of manifold diseases as a novel subtype of non-coding RNAs. Cheng et al (40) were the first to demonstrate that circVMA21 derived from vacuolar ATPase assembly factor gene is markedly decreased in the degenerative NP specimens compared with the normal NP tissues based on RT-qPCR analyses. Functionally, circVMA21 is able to protect against IVDD by suppressing inflammatory cytokine-induced NP cell apoptosis, downregulating the expression of catabolic enzymes (MMP-3, MMP-13, ADAMTS4 and ADAMTS5) and promoting synthesis of ECM. Mechanistically, circVMA21 is expected to function as ceRNAs to modulate the pathological process of IVDD through sponging miR-200c and targeting X-linked inhibitor-of-apoptosis protein. Recently, Wang et al (89) analyzed the expression profiling of human lumbar disc circRNAs based on an online database and reported that circSEMA4B is substantially downregulated in degenerative lumbar disc tissues. Functionally, circSEMA4B can inhibit the development of IVDD by enhancing NP cell proliferation and alleviating cell senescence and ECM degradation. Mechanistically, circSEMA4B is a potential therapeutic target for IVDD as it represses miR-431 via the Wnt/ $\beta$-catenin signaling pathway (89). However, circRNA_104670 and circRNA_0058097 are upregulated in degenerative NP tissues and tension-induced degenerative endplate chondrocytes, and it has been reported that they promote the progression of IVDD by acting as ceRNAs $(41,90)$. Furthermore, Song et al (41) confirmed via the dual-luciferase and EGFP/RFP reporter assays that circRNA_104670 directly binds to miR-17-3p, while MMP-2 is the direct target of miR-17-3p. Knockdown and overexpression of circRNA 104670 was followed by the 
Table II. Characteristics of lncRNAs involved in the process of intervertebral disc degeneration.

\begin{tabular}{|c|c|c|c|c|c|c|}
\hline lncRNAs & Specimen & Expression & Target & Function & Author, year & (Refs.) \\
\hline SNHG1 & NP & Up & miR-326 & Proliferation $\uparrow$ & Tan et al, 2018 & (39) \\
\hline HOTAIR & NP & Down & miR-34a & Apoptosis $\downarrow$ & Yu et al, 2018 & (76) \\
\hline lncPolE & NP & Up & PolE1 & Apoptosis $\uparrow$ & Li et al, 2019 & $(77)$ \\
\hline GAS5 & NP & Up & miR-155 & Apoptosis $\uparrow$ & Wang et al, 2019 & (78) \\
\hline LINC00969 & NP & Up & miR-335-3p & Apoptosis $\uparrow$ & Yu et al, 2019 & (79) \\
\hline HCG18 & NP & Up & miR-146a-5p & Apoptosis $\uparrow$ & Xi et al, 2017 & $(80)$ \\
\hline RMRP & NP & Up & miR-206 & $\begin{array}{l}\text { Proliferation } \uparrow, \\
\text { ECM-degradation } \uparrow\end{array}$ & Wang et al, 2018 & $(81)$ \\
\hline FAM83H-AS1 & NP & Up & Notch 1 & $\begin{array}{l}\text { Proliferation } \uparrow, \\
\text { ECM-degradation } \uparrow\end{array}$ & Wei et al, 2019 & $(82)$ \\
\hline NEAT1 & NP & $\mathrm{Up}$ & ERK/MAPK & ECM-degradation $\uparrow$ & Ruan et al, 2018 & $(83)$ \\
\hline linc-ADAMTS5 & NP & Down & ADAMTS5 & ECM-degradation $\downarrow$ & Wang et al, 2017 & $(84)$ \\
\hline TUG1 & NP & Up & Wnt $\beta$-catenin & $\begin{array}{l}\text { Senescence } \uparrow, \\
\text { ECM-degradation } \uparrow, \\
\text { Apoptosis } \uparrow\end{array}$ & Chen et al, 2017 & $(85)$ \\
\hline H19 & NP & Up & miR-22 & $\begin{array}{l}\text { Senescence } \uparrow, \\
\text { ECM-degradation } \uparrow\end{array}$ & Wang et al, 2018 & $(86)$ \\
\hline LINC00641 & NP & Up & miR-153-3 & Autophagy $\uparrow$ & Wang et al, 2019 & $(87)$ \\
\hline \multicolumn{7}{|c|}{$\begin{array}{l}\uparrow \text {, promotion; } \downarrow \text {, inhibition. lncRNAs, long non-coding RNAs; ECM, extracellular matrix; NP, nucleus pulposus; SNHG1, small nucleolat } \\
\text { RNA host gene 1; HOTAIR, homeobox transcript antisense intergenic RNA; GAS5, growth arrest specific 5; LINC00969, long intergenic } \\
\text { non-protein coding RNA 969; HCG18, HLA complex group 18; RMRP, RNA component of mitochondrial RNA processing endoribonuclease } \\
\text { FAM83H-AS1, IQ motif and ankyrin repeat containing; NEAT1, nuclear paraspeckle assembly transcript 1; linc, long intergenic non-protein } \\
\text { coding RNA; ADAMTS5, A disintegrin and metalloproteinase with thrombospondin motif 5; TUG1, taurine upregulated gene 1; H19, H19 } \\
\text { imprinted maternally expressed transcript; LINC00641, long intergenic non-protein coding RNA 641; PolE1, DNA polymerase E catalytic } \\
\text { subunit A; miR, microRNA; MAPK, mitogen-activated protein kinase. }\end{array}$} \\
\hline
\end{tabular}

Table III. Characteristics of circRNAs involved in the process of intervertebral disc degeneration.

\begin{tabular}{|c|c|c|c|c|c|c|}
\hline circRNA & Specimen & Expression & Target & Function & Author, year & (Refs.) \\
\hline circVMA21 & NP & Down & $\mathrm{miR}-200 \mathrm{c}$ & $\begin{array}{l}\text { Apoptosis } \downarrow \text {, } \\
\text { ECM-degradation } \downarrow\end{array}$ & Cheng et al, 2018 & $(40)$ \\
\hline circRNA_104670 & NP & Up & $\operatorname{miR}-17-3 p$ & $\begin{array}{l}\text { Apoptosis } \uparrow, \\
\text { Proliferation } \downarrow \text {, } \\
\text { ECM-degradation } \uparrow\end{array}$ & Song et al, 2018 & $(41)$ \\
\hline circSEMA4B & NP & Down & $\operatorname{miR}-431$ & $\begin{array}{l}\text { Proliferation } \uparrow \text {, } \\
\text { ECM-degradation } \downarrow \text {, } \\
\text { Senescence } \downarrow\end{array}$ & Wang et al, 2018 & $(89)$ \\
\hline circRNA_0058097 & CEP & $\mathrm{Up}$ & $\operatorname{miR}-365 a-5 p$ & ECM-degradation $\uparrow$ & Xiao et al, 2019 & $(90)$ \\
\hline
\end{tabular}

$\uparrow$, promotion; $\downarrow$, inhibition. circRNA, circular RNA; ECM, extracellular matrix; NP, nucleus pulposus; CEP, cartilage endplates; miR, microRNA.

observation of proliferation and apoptosis of NP cells and the expression of miR-17-3p and ECM-related gene compared with the control group. Functionally, through gain- and loss-of-function studies, circRNA_104670 was demonstrated to inhibit proliferation of NP cells and expression of collagen II, and promote apoptosis and the expression of MMP-2 by targeting miR-17-3p and MMP-2. Xiao et al (90) reported that circRNA_0058097 may promote morphological changes of endplate chondrocytes and enhance ECM degradation and degeneration of IVDs by upregulating the expression of histone deacetylase 4 through sponging miR-365a-5p. Thus, circRNA_0058097 promotes the pathological process of 
IVDD by regulating tension-induced degeneration of endplate chondrocytes. CircRNAs modulate the development of IVDD by functioning as ceRNAs (90) and may serve as a potential novel therapeutic target of IVDD, similar to miRNAs and lncRNAs (Fig. 5).

\section{Conclusions}

As one of the most prevalent diseases among the elderly population, NLBP has caused tremendous pressure on medical resources and the economy. Several studies have demonstrated that IVDD is responsible for the pathogenesis of NLBP; however, its underlying molecular and cellular mechanisms remain unclear. Recently, the role of non-coding RNAs in several diseases emerged, including IVDD.

In the present review, the role of miRNAs, IncRNAs and circRNAs in the progression of IVDD is summarized. Furthermore, it presents a summary of how to modulate the proliferation, senescence, apoptosis and ECM degradation of NP and CEP by regulating downstream target genes (Tables I-III). The data presented in the current review provide novel insights into the etiology of IVDD and identifies non-coding RNAs as a potential novel target for the treatment of IVDD. However, there is still a lack of relevant studies on miRNAs and circRNAs as therapeutic targets for IVDD. With the development of nanoparticle technology and an in-depth understanding of the pathogenesis of IVDD, research on non-coding RNAs, particularly miRNAs, IncRNAs and circRNAs as therapeutic targets for the treatment of IVDD have potential to become a novel research focus.

\section{Acknowledgements}

Not applicable.

\section{Funding}

The present review was supported by The National Natural Science Foundation of China (NSFC; grant no. 31971275).

\section{Availability of data and materials}

Not applicable.

\section{Authors' contributions}

HW and LW designed the present review. JJ, YS and GX performed the literature review and drafted the initial manuscript. HW and LW critically revised the manuscript for important intellectual content. All authors have read and approved the manuscript.

\section{Ethics approval and consent to participate}

Not applicable.

\section{Patient consent for publication}

Not applicable.

\section{Competing interests}

The authors declare that they have no competing interests.

\section{References}

1. Maher C, Underwood M and Buchbinder R: Non-specific low back pain. Lancet 389: 736-747, 2017.

2. Dudli S, Fields AJ, Samartzis D, Karppinen J and Lotz JC: Pathobiology of modic changes. Eur Spine J 25: 3723-3734, 2016.

3. Vos T, Flaxman AD, Naghavi M, Lozano R, Michaud C, Ezzati M, Shibuya K, Salomon JA, Abdalla S, Aboyans V, et al: Years lived with disability (YLDs) for 1160 sequelae of 289 diseases and injuries 1990-2010: A systematic analysis for the global burden of disease study 2010. Lancet 380: 2163-2196, 2012.

4. Hartvigsen J, Hancock MJ, Kongsted A, Louw Q, Ferreira ML, Genevay S, Hoy D, Karppinen J, Pransky G, Sieper J, et al: What low back pain is and why we need to pay attention. Lancet 391: 2356-2367, 2018

5. Yelin E, Weinstein S and King T: The burden of musculoskeletal diseases in the United States. Semin Arthritis Rheum 46: 259-260, 2016.

6. Foster NE, Anema JR, Cherkin D, Chou R, Cohen SP, Gross DP, Ferreira PH, Fritz JM, Koes BW, Peul W, et al: Prevention and treatment of low back pain: Evidence, challenges, and promising directions. Lancet 391: 2368-2383, 2018.

7. Risbud MV and Shapiro IM: Role of cytokines in intervertebral disc degeneration: Pain and disc content. Nat Rev Rheumatol 10: 44-56, 2014.

8. Adams MA and Roughley PJ: What is intervertebral disc degeneration, and what causes it? Spine (Phila Pa 1976) 31: 2151-2161, 2006.

9. Vo NV, Hartman RA, Patil PR, Risbud MV, Kletsas D, Iatridis JC, Hoyland JA, Le Maitre CL, Sowa GA and Kang JD: Molecular mechanisms of biological aging in intervertebral discs. J Orthop Res 34: 1289-1306, 2016.

10. Bian Q, Jain A, Xu X, Kebaish K, Crane JL, Zhang Z, Wan M, Ma L, Riley LH, Sponseller PD, et al: Excessive activation of TGF $\beta$ by spinal instability causes vertebral endplate sclerosis. Sci Rep 6: 27093, 2016.

11. Huang YC, Urban JP and Luk KD: Intervertebral disc regeneration: Do nutrients lead the way? Nat Rev Rheumatol 10: 561-566, 2014.

12. Liao Z, Wu X, Song Y, Luo R, Yin H, Zhan S, Li S, Wang K, Zhang Y and Yang C: Angiopoietin-like protein 8 expression and association with extracellular matrix metabolism and inflammation during intervertebral disc degeneration. J Cell Mol Med 23: 5737-5750, 2019.

13. Liu X, Zhuang J, Wang D, Lv L, Zhu F, Yao A and Xu T: Glycyrrhizin suppresses inflammation and cell apoptosis by inhibition of HMGB1 via p38/p-JUK signaling pathway in attenuating intervertebral disc degeneration. Am J Transl Res 11: 5105-5113, 2019.

14. Grant MP, Epure LM, Bokhari R, Roughley P, Antoniou J and Mwale F: Human cartilaginous endplate degeneration is induced by calcium and the extracellular calcium-sensing receptor in the intervertebral disc. Eur Cell Mater 32: 137-151, 2016.

15. Yao Y, Deng Q, Song W, Zhang H, Li Y, Yang Y, Fan X, Liu M, Shang J, Sun C, et al: MIF plays a key role in regulating tissue-specific chondro-osteogenic differentiation fate of human cartilage endplate stem cells under hypoxia. Stem Cell Reports 7: 249-262, 2016.

16. Tang Z, Hu B, Zang F, Wang J, Zhang X and Chen H: Nrf2 drives oxidative stress-induced autophagy in nucleus pulposus cells via a Keap1/Nrf2/p62 feedback loop to protect intervertebral disc from degeneration. Cell Death Dis 10: 510, 2019.

17. Wang G, Huang K, Dong Y, Chen S, Zhang J, Wang J, Xie Z, Lin X, Fang $X$ and Fan S: Lycorine suppresses endplate-chondrocyte degeneration and prevents intervertebral disc degeneration by inhibiting NF- $\kappa \mathrm{B}$ signalling pathway. Cell Physiol Biochem 45: 1252-1269, 2018.

18. Matsui M and Corey DR: Non-coding RNAs as drug targets. Nat Rev Drug Discov 16: 167-179, 2017.

19. Ning B, Yu D and Yu AM: Advances and challenges in studying noncoding RNA regulation of drug metabolism and development of RNA therapeutics. Biochem Pharmacol 169: 113638, 2019. 
20. Wang WT, Han C, Sun YM, Chen TQ and Chen YQ: Noncoding RNAs in cancer therapy resistance and targeted drug development. J Hematol Oncol 12: 55, 2019.

21. Guttman M and Rinn JL: Modular regulatory principles of large non-coding RNAs. Nature 482: 339-346, 2012.

22. Sato-Kuwabara Y, Melo SA, Soares FA and Calin GA: The fusion of two worlds: Non-coding RNAs and extracellular vesicles-diagnostic and therapeutic implications (Review). Int J Oncol 46: 17-27, 2015

23. Zhou X, Chen L, Grad S, Alini M, Pan H, Yang D, Zhen W, Li Z, Huang S and Peng S: The roles and perspectives of microRNAs as biomarkers for intervertebral disc degeneration. J Tissue Eng Regen Med 11: 3481-3487, 2017.

24. St Laurent G, Wahlestedt C and Kapranov P: The Landscape of long noncoding RNA classification. Trends Genet 31: 239-251, 2015.

25. Memczak S, Jens M, Elefsinioti A, Torti F, Krueger J, Rybak A, Maier L, Mackowiak SD, Gregersen LH, Munschauer M, et al: Circular RNAs are a large class of animal RNAs with regulatory potency. Nature 495: 333-338, 2013.

26. Zhou L, Dong S, Deng Y, Yang P, Zheng Y, Yao L, Zhang M, Yang S, Wu Y, Zhai Z, et al: GOLGA7 rs11337, a polymorphism at the MicroRNA binding site, is associated with glioma prognosis. Mol Ther Nucleic Acids 18: 56-65, 2019.

27. Bhan A, Soleimani M and Mandal SS: Long noncoding RNA and cancer: A new paradigm. Cancer Res 77: 3965-3981, 2017.

28. Wei Y, Chen X, Liang C, Ling Y, Yang X, Ye X, Zhang H, Yang P, Cui X, Ren Y, et al: A noncoding regulatory RNAs network driven by Circ-CDYL acts specifically in the early stages hepatocellular carcinoma. Hepatology 71: 130-147, 2020.

29. Shang Q, Yang Z, Jia R and Ge S: The novel roles of circRNAs in human cancer. Mol Cancer 18: 6, 2019.

30. Wu K, Liao X, Gong Y, He J, Zhou JK, Tan S, Pu W, Huang C, Wei YQ and Peng Y: Circular RNA F-circSR derived from SLC34A2-ROS1 fusion gene promotes cell migration in non-small cell lung cancer. Mol Cancer 18: 98, 2019.

31. Wu Y, Xie Z, Chen J, Chen J, Ni W, Ma Y, Huang K, Wang G, Wang J, Ma J, et al: Circular RNA circTADA2A promotes osteosarcoma progression and metastasis by sponging miR-203a-3p and regulating CREB3 expression. Mol Cancer 18: $73,2019$.

32. Lange S, Banerjee I, Carrion K, Serrano R, Habich L, Kameny R, Lengenfelder L, Dalton N, Meili R, Börgeson E, et al: miR-486 is modulated by stretch and increases ventricular growth. JCI Insight 4: e125507, 2019.

33. Calderon-Dominguez M, Belmonte T, Quezada-Feijoo M, Ramos-Sánchez M, Fernández-Armenta J, Pérez-Navarro A, Cesar S, Peña-Peña L, Vea À, Llorente-Cortés V, et al: Emerging role of microRNAs in dilated cardiomyopathy: Evidence regarding etiology. Transl Res 215: 86-101, 2020.

34. Cai B, Zhang Y, Zhao Y, Wang J, Li T, Zhang Y, Jiang Y, Jin X, Xue G, Li P, et al: Long noncoding RNA-DACH1 (Dachshund Homolog 1) regulates cardiac function by inhibiting SERCA2a (Sarcoplasmic Reticulum Calcium ATPase 2a). Hypertension 74 833-842, 2019

35. Huang S, Li X, Zheng H, Si X, Li B, Wei G, Li C, Chen Y, Chen Y, Liao W, et al: Loss of super-enhancer-regulated circRNA Nfix induces cardiac regeneration after myocardial infarction in adult mice. Circulation 139: 2857-2876, 2019.

36. Hasvik E, Schjølberg T, Jacobsen DP, Haugen AJ, Grøvle L, Schistad EI and Gjerstad J: Up-regulation of circulating microRNA-17 is associated with lumbar radicular pain following disc herniation. Arthritis Res Ther 21: 186, 2019.

37. Wang C, Zhang ZZ, Yang W, Ouyang ZH, Xue JB, Li XL, Zhang J, Chen WK, Yan YG and Wang WJ: MiR-210 facilitates ECM degradation by suppressing autophagy via silencing of ATG7 in human degenerated NP cells. Biomed Pharmacother 93: 470-479, 2017

38. Shao T, Hu Y, Tang W, Shen H, Yu Z and Gu J: The long noncoding RNA HOTAIR serves as a microRNA-34a-5p sponge to reduce nucleus pulposus cell apoptosis via a NOTCH1-mediated mechanism. Gene 715: 144029, 2019.

39. Tan H, Zhao L, Song R, Liu Y and Wang L: The long noncoding RNA SNHG1 promotes nucleus pulposus cell proliferation through regulating miR-326 and CCND1. Am J Physiol Cell Physiol 315: C21-C27, 2018.

40. Cheng X, Zhang L, Zhang K, Zhang G, Hu Y, Sun X, Zhao C, $\mathrm{Li} \mathrm{H}, \mathrm{Li}$ YM and Zhao J: Circular RNA VMA21 protects against intervertebral disc degeneration through targeting miR-200c and X linked inhibitor-of-apoptosis protein. Ann Rheum Dis 77 : $770-779,2018$
41. Song J, Wang HL, Song KH, Ding ZW, Wang HL, Ma XS, Lu FZ, Xia XL, Wang YW, Fei-Zou and Jiang JY: CircularRNA_104670 plays a critical role in intervertebral disc degeneration by functioning as a ceRNA. Exp Mol Med 50: 94, 2018.

42. Lu TX and Rothenberg ME: MicroRNA. J Allergy Clin Immunol 141: 1202-1207, 2018

43. Wang B, Wang D, Yan T and Yuan H: miR-138-5p promotes TNF- $\alpha$-induced apoptosis in human intervertebral disc degeneration by targeting SIRT1 through PTEN/PI3K/Akt signaling. Exp Cell Res 345: 199-205, 2016.

44. Hayes J, Peruzzi PP and Lawler S: MicroRNAs in cancer: Biomarkers, functions and therapy. Trends Mol Med 20: 460-469, 2014.

45. Wang C, Wang WJ, Yan YG, Xiang YX, Zhang J, Tang ZH and Jiang ZS: MicroRNAs: New players in intervertebral disc degeneration. Clin Chim Acta 450: 333-341, 2015.

46. Mo YY: MicroRNA regulatory networks and human disease. Cell Mol Life Sci 69: 3529-3531, 2012.

47. Ivey KN and Srivastava D: microRNAs as developmental regulators. Cold Spring Harb Perspect Biol 7: a008144, 2015.

48. Bartel DP: MicroRNAs: Target recognition and regulatory functions. Cell 136: 215-233, 2009

49. Liu B, Li J and Cairns MJ: Identifying miRNAs, targets and functions. Brief Bioinform 15: 1-19, 2014.

50. Chi Y, Wang D, Wang J, Yu W and Yang J: Long non-coding RNA in the pathogenesis of cancers. Cells 8: 1015, 2019.

51. Robinson EK, Covarrubias S and Carpenter S: The how and why of lncRNA function: An innate immune perspective. Biochim Biophys Acta Gene Regul Mech 1863: 194419, 2019.

52. Ji E, Kim C, Kim W and Lee EK: Role of long non-coding RNAs in metabolic control. Biochim Biophys Acta Gene Regul Mech 1863: 194348, 2020

53. Chen WK, Yu XH, Yang W, Wang C, He WS, Yan YG, Zhang J and Wang WJ: lncRNAs: Novel players in intervertebral disc degeneration and osteoarthritis. Cell Prolif 50: e12313, 2017.

54. Ulitsky I and Bartel DP: lincRNAs: Genomics, evolution, and mechanisms. Cell 154: 26-46, 2013.

55. Yang L, Lin C, Jin C, Yang JC, Tanasa B, Li W, Merkurjev D, Ohgi KA, Meng D, Zhang J, et al: lncRNA-dependent mechanisms of androgen-receptor-regulated gene activation programs. Nature 500: 598-602, 2013.

56. Santer L, Bär C and Thum T: Circular RNAs: A novel class of functional RNA molecules with a therapeutic perspective. Mol Ther 27: 1350-1363, 2019.

57. Suzuki H,Zuo Y, Wang J,Zhang MQ, Malhotra A and Mayeda A: Characterization of RNase R-digested cellular RNA source that consists of lariat and circular RNAs from pre-mRNA splicing. Nucleic Acids Res 34: e63, 2006.

58. Jeck WR and Sharpless NE: Detecting and characterizing circular RNAs. Nat Biotechnol 32: 453-461, 2014.

59. Kristensen LS, Andersen MS, Stagsted LVW, Ebbesen KK, Hansen TB and Kjems J: The biogenesis, biology and characterization of circular RNAs. Nat Rev Genet 20: 675-691, 2019.

60. Li X, Yang L and Chen LL: The biogenesis, functions, and challenges of circular RNAs. Mol Cell 71: 428-442, 2018.

61. Szabo L and Salzman J: Detecting circular RNAs: Bioinformatic and experimental challenges. Nat Rev Genet 17: 679-692, 2016.

62. Li W, Wang P, Zhang Z, Wang W, Liu Y and Qi Q: miR-184 regulates proliferation in nucleus pulposus cells by targeting GAS1. World Neurosurg 97: 710-715.e1, 2017.

63. Che YJ, Guo JB, Liang T, Chen X, Zhang W, Yang HL and Luo ZP: Assessment of changes in the micro-nano environment of intervertebral disc degeneration based on Pfirrmann grade. Spine J 19: 1242-1253, 2019.

64. Yang SD, Yang DL, Sun YP, Wang BL, Ma L, Feng SQ and Ding WY: $17 \beta$-estradiol protects against apoptosis induced by interleukin- $1 \beta$ in rat nucleus pulposus cells by down-regulating MMP-3 and MMP-13. Apoptosis 20: 348-357, 2015

65. Wang T, Li P, Ma X, Tian P, Han C, Zang J, Kong J and Yan H: MicroRNA-494 inhibition protects nucleus pulposus cells from TNF- $\alpha$-induced apoptosis by targeting JunD. Biochimie 115: 1-7, 2015.

66. Wang WJ, Yang W, Ouyang ZH, Xue JB, Li XL, Zhang J, He WS, Chen WK, Yan YG and Wang C: MiR-21 promotes ECM degradation through inhibiting autophagy via the PTEN/akt/mTOR signaling pathway in human degenerated NP cells. Biomed Pharmacother 99: 725-734, 2018

67. Zhao B, Yu Q, Li H, Guo X and He X: Characterization of microRNA expression profiles in patients with intervertebral disc degeneration. Int J Mol Med 33: 43-50, 2014. 
68. Ji ML, Zhang XJ, Shi PL, Lu J, Wang SZ, Chang Q, Chen H and Wang C: Downregulation of microRNA-193a-3p is involved in invertebral disc degeneration by targeting MMP14. J Mol Med (Berl) 94: 457-468, 2016.

69. Xu YQ, Zhang ZH, Zheng YF and Feng SQ: Dysregulated miR-133a mediates loss of type II collagen by directly targeting matrix metalloproteinase 9 (MMP9) in human intervertebra disc degeneration. Spine (Phila Pa 1976) 41: E717-E724, 2016.

70. Hua WB, Wu XH, Zhang YK, Song Y, Tu J, Kang L, Zhao KC, Li S, Wang K, Liu W, et al: Dysregulated miR-127-5p contributes to type II collagen degradation by targeting matrix metalloproteinase-13 in human intervertebral disc degeneration. Biochimie 139: 74-80, 2017.

71. Ji ML, Lu J, Shi PL, Zhang XJ, Wang SZ, Chang Q, Chen H and Wang C: Dysregulated miR-98 contributes to extracellular matrix degradation by targeting IL-6/STAT3 signaling pathway in human intervertebral disc degeneration. J Bone Miner Res 31 : 900-909, 2016.

72. Chen H, Wang J, Hu B, Wu X, Chen Y, Li R and Yuan W: miR-34a promotes Fas-mediated cartilage endplate chondrocyte apoptosis by targeting Bcl-2. Mol Cell Biochem 406: 21-30, 2015.

73. Liu MH, Sun C, Yao Y, Fan X, Liu H, Cui YH, Bian XW, Huang B and Zhou Y: Matrix stiffness promotes cartilage endplate chondrocyte calcification in disc degeneration via miR-20a targeting ANKH expression. Sci Rep 6: 25401, 2016

74. Xiao L, Xu S, Xu Y, Liu C, Yang B, Wang J and $\mathrm{Xu} \mathrm{H}$ : TGF- $\beta$ /SMAD signaling inhibits intermittent cyclic mechanical tension-induced degeneration of endplate chondrocytes by regulating the miR-455-5p/RUNX2 axis. J Cell Biochem 119: 10415-10425, 2018.

75. Li Z, Li X, Chen C, Li S, Shen J, Tse G, Chan MTV and Wu WKK: Long non-coding RNAs in nucleus pulposus cell function and intervertebral disc degeneration. Cell Prolif 51: e12483, 2018

76. Yu Y, Zhang X, Li Z, Kong L and Huang Y: LncRNA HOTAIR suppresses TNF- $\alpha$ induced apoptosis of nucleus pulposus cells by regulating miR-34a/Bcl-2 axis. Biomed Pharmacother 107: 729-737, 2018

77. Li X, Lou Z, Liu J, Li H, Lei Y,Zhao X and Zhang F: Upregulation of the long noncoding RNA lncPolE contributes to intervertebra disc degeneration by negatively regulating DNA polymerase epsilon. Am J Transl Res 11: 2843-2854, 2019.

78. Wang Y, Song Q, Huang X, Chen Z, Zhang F, Wang K, Huang G and Shen H: Long noncoding RNA GAS5 promotes apoptosis in primary nucleus pulposus cells derived from the human intervertebral disc via Bcl-2 downregulation and caspase 3 upregulation. Mol Med Rep 19: 2164-2172, 2019.

79. Yu L, Hao Y, Xu C, Zhu G and Cai Y: LINC00969 promotes the degeneration of intervertebral disk by sponging miR-335-3p and regulating NLRP3 inflammasome activation. IUBMB life 71: 611-618, 2019.
80. Xi Y, Jiang T, Wang W, Yu J, Wang Y, Wu X and He Y: Long non-coding HCG18 promotes intervertebral disc degeneration by sponging miR-146a-5p and regulating TRAF6 expression. Sci Rep 7: 13234, 2017.

81. Wang X, Peng L, Gong X, Zhang X, Sun R and Du J: lncRNA-RMRP promotes nucleus pulposus cell proliferation through regulating miR-206 expression. J Cell Mol Med 22: 5468-5476, 2018.

82. Wei R, Chen Y, Zhao Z, Gu Q and Wu J: LncRNA FAM83H-AS1 induces nucleus pulposus cell growth via targeting the Notch signaling pathway. J Cell Physiol 234: 22163-22171, 2019.

83. Ruan Z, Ma H, Li J, Liu H, Jia H and Li F: The long non-coding RNA NEAT1 contributes to extracellular matrix degradation in degenerative human nucleus pulposus cells. Exp Biol Med (Maywood) 243: 595-600, 2018.

84. Wang K, Song Y, Liu W, Wu X, Zhang Y, Li S, Kang L, Tu J, Zhao K, Hua W and Yang C: The noncoding RNA linc-ADAMTS5 cooperates with RREB1 to protect from intervertebral disc degeneration through inhibiting ADAMTS5 expression. Clin Sci (Lond) 131: 965-979, 2017.

85. Chen J, Jia YS, Liu GZ, Sun Q, Zhang F, Ma S and Wang YJ: Role of LncRNA TUG1 in intervertebral disc degeneration and nucleus pulposus cells via regulating Wnt/ $\beta$-catenin signaling pathway. Biochem Biophys Res Commun 491: 668-674, 2017.

86. Wang X, Zou M, Li J, Wang B, Zhang Q, Liu F and Lü G: IncRNA $\mathrm{H} 19$ targets miR-22 to modulate $\mathrm{H}_{2} \mathrm{O}_{2}$-induced deregulation in nucleus pulposus cell senescence, proliferation, and ECM synthesis through Wnt signaling. J Cell Biochem 119: 4990-5002, 2018.

87. Wang XB, Wang H, Long HQ, Li DY and Zheng X: LINC00641 regulates autophagy and intervertebral disc degeneration by acting as a competitive endogenous RNA of miR-153-3p under nutrition deprivation stress. J Cell Physiol 234: 7115-7127, 2019.

88. Sampara P, Banala RR, Vemuri SK, Av GR and Gpv S: Understanding the molecular biology of intervertebral disc degeneration and potential gene therapy strategies for regeneration: A review. Gene Ther 25: 67-82, 2018.

89. Wang X, Wang B, Zou M, Li J, Lü G, Zhang Q, Liu F and Lu C: CircSEMA4B targets miR-431 modulating IL-1 $\beta$-induced degradative changes in nucleus pulposus cells in intervertebral disc degeneration via Wnt pathway. Biochim Biophys Acta Mol Basis Dis 1864: 3754-3768, 2018

90. Xiao L, Ding B, Xu S, Gao J, Yang B, Wang J and Xu H: circRNA_0058097 promotes tension-induced degeneration of endplate chondrocytes by regulating HDAC4 expression through sponge adsorption of miR-365a-5p. J Cell Biochem 121: 418-429, 2019.

c) (7) $\Theta$ This work is licensed under a Creative Commons Attribution-NonCommercial-NoDerivatives 4.0 International (CC BY-NC-ND 4.0) License. 